\title{
Oral Lesions: Poor Markers of Virologic Failure in HIV-Infected Patients on Antiretroviral Therapy
}

\author{
Irene Tami-Maury, ${ }^{1,2}$ James Willig, ${ }^{3}$ Pauline Jolly, ${ }^{1}$ Sten H. Vermund, ${ }^{4}$ Inmaculada Aban, ${ }^{5}$ \\ Jeff Hill, ${ }^{6}$ and Craig M. Wilson ${ }^{1,7}$
}

${ }^{1}$ Department of Epidemiology, School of Public Health, University of Alabama at Birmingham, Birmingham, AL 35294, USA

${ }^{2}$ Department of Behavioral Science, The University of Texas MD Anderson Cancer Center, 1155 Pressler St., Unit 1330, CPB3.3202, Houston, TX 77030-4009, USA

${ }^{3}$ Department of Infectious Diseases, School of Medicine, University of Alabama at Birmingham, Birmingham, AL 35294, USA

${ }^{4}$ Vanderbilt Institute for Global Health, Vanderbilt University, Nashville, TN 37203, USA

${ }^{5}$ Department of Biostatistics, School of Public Health, University of Alabama at Birmingham, Birmingham, AL 35294, USA

${ }^{6}$ Department of Predoctoral General Dentistry, School of Dentistry, University of Alabama at Birmingham, Birmingham, AL 35294, USA

${ }^{7}$ Sparkman Center for Global Health, University of Alabama at Birmingham, Birmingham, AL 35294, USA

Correspondence should be addressed to Irene Tami-Maury; irene.tami@fulbrightmail.org

Received 18 September 2012; Accepted 14 October 2012

Academic Editors: G. A. Funk and G. Simo

Copyright (C) 2013 Irene Tami-Maury et al. This is an open access article distributed under the Creative Commons Attribution License, which permits unrestricted use, distribution, and reproduction in any medium, provided the original work is properly cited.

The objective of this study was to assess the use of HIV-oral lesions (OLs) as markers of virologic failure (VF) in response to antiretroviral therapy (ART). Concurrent virologic status was compared between 744 individuals with and without OL $\left(X^{2}\right)$. Time to VF between OL groups was compared (Kaplan-Meier), and baseline factors associated with VF were determined (Cox Hazard models). Sensitivity, specificity, positive predictive value, and negative predictive value were also computed. At baseline and 12 months, individuals with OL were more likely to have viral load $\geq 10,000$ copies $/ \mathrm{ml}$ compared to patients without OL $(P<0.01)$. Time to VF between patients with baseline OL (mean: 17 months, 95\% CI 16-18) and patients without baseline OL (mean: 19 months, 95\% CI: 18-20) was statistically different. Patients who were African-American (HR 1.356; 95\% CI: 1.045-0.759), ARTexperienced (HR 2.298; 95\% CI: 1.743-3.030), had mental disorders (HR 1.410; 95\% CI: 1.078-1.843), and had high baseline viral load (HR 2.82; 95\% CI: 1.661-3.137), were more likely to have VF after the first six months of ART. OL had a moderate positive predictive value for concurrent VF at 6 months (45.5\%) and 18 months (33.3\%), but a strong positive predictive value at 12 months $(80.0 \%)$ and 24 months (100.0\%). Findings of this study suggest that OLs could be poor predictors of VF in HIV-infected patients on ART therapy.

\section{Introduction}

Since the mid-1980s there has been an intensive search for clinical markers to assess and monitor disease progression from initial HIV infection to the development of acquired immunodeficiency syndrome (AIDS). Oral manifestations of patients with HIV infection have been considered to be of particular value in this regard [1-3]. Moreover, persistent candidiasis and hairy leukoplakia, in particular, are strongly associated with immunologic failure and progression of HIV disease $[4,5]$.

The use of antiretroviral therapy (ART) has produced important therapeutic effects and dramatic changes in the clinical prognosis of HIV infection. ART improves the quality of life of the HIV-infected patient by restraining viral replication and allowing the immune system to replenish the destroyed CD4+ T cells (CD4) $[6,7]$. The amount of CD4 per milliliter and/or the quantity of RNA-HIV-1 copies 
per milliliter-viral load-are surrogate markers of HIV disease status and progression. While the CD4 counts are used to determine the risk of opportunistic illnesses, assess prognosis, and guide decisions about when to start ART therapy, viral load tests are critical in monitoring virologic response to ART therapy.

More research is needed before using clinical markers in deciding when to initiate or switch ART when CD4 cell counts or viral load testing are not available. Although related data exist, the true nature of the association of oral lesions (OLs) and monitoring measurements (especially viral load) is not completely clear [8-10].

OLs have been used as clinical criteria for the WHO classification system in adults and adolescents with HIV infection [11]. Neither angular cheilitis (clinical stage 2), oral candidiasis (clinical stage 3) nor oral hairy leukoplakia (clinical stage 3), are by themselves staging events that impact therapy decisions according to the WHO guidelines. However, the presence of oral candidiasis and oral hairy leukoplakia, in combination with a baseline CD4 cell count below 200 cells $/ \mathrm{mm}^{3}$, may guide the decision to initiate or change ART.

Therefore, the first aim of this pilot study was to investigate the association between OL and the concurrent virologic failure (VF) after initiating ART among HIV/AIDS patients at an HIV Outpatient Clinic in Birmingham, AL, USA. The second aim was to assess time to VF after the first six months of therapy and during the 24-month follow-up period. The third aim was to determine the baseline factors associated with time to VF, especially, presence/absence of baseline OL. The fourth and final aim was to assess the sensitivity, specificity, positive predictive value, and negative predictive value of $\mathrm{OL}$ as a marker for concurrent $\mathrm{VF}$ at subsequent predefined time points after the first six months of therapy. The UAB Institutional Review Board approved this protocol.

\section{Materials and Methods}

2.1. Study Population. The 1917 Clinic Cohort is an ongoing single-center, longitudinal HIV observational study established in 1992 in the University of Alabama at Birmingham (UAB). To be included in this retrospective study, based on chart review, the patient had to be at least 19 years old with known HIV infection, entered the 1917 Clinic for the first time between January 2000 and June 2006, initiating ART, and had at least 4 clinic visits within the 2 -year followup period. The following information was obtained: date of birth, sex, race/ethnicity, insurance status, transmission risk category, baseline $\mathrm{CD} 4$ and viral load in a range of 120 days prior and 30 days after the initial 1917 Clinic care visit, and CD4 and viral load every 6 months after the first six months of therapy over the 24-month followup period. ART naïve patients (those individuals newly diagnosed with HIV infection and initiating ART for the first time) and ART-experienced patients (individuals previously exposed to ART and initiating a new regimen at the 1917 Clinic) were included in the study sample. At time of first presentation at the clinic, all patients (both ART-naïve and ART-experienced) had a "baseline visit" for structured interviewed and clinical/laboratory examination. Afterwards they were appointed for scheduled visits to monitoring ART therapy.

\subsection{Operational Definitions}

2.2.1. Oral Lesion $(O L)$. The main predictor variable was presence or absence of any OL at baseline, 6, 12, 18, and 24 months. OL clinical assessment and recording was performed by clinical site staff and reviewed by the attending physician. OLs were classified according to the diagnostic criteria of the Clearinghouse on Oral Problems Related to HIV Infection and WHO Collaborating Centre on Oral Manifestations of the Immunodeficiency Virus (Table 1). OL was dichotomized into yes or no. Even though there is a diverse pathophysiology of the OL studied, it was decided to aggregate them into a single analytical variable for clinical and epidemiological purposes, since in the HIV care settings, especially in the developing world, the treating general physicians without dental/oral training are the ones performing intraoral examination of the patients most of the time.

All clinical presentations of candidiasis (erythematous candidiasis, pseudomembranous candidiasis, chronic hyperplastic candidiasis, angular cheilitis) were grouped as oropharyngeal candidiasis (OPC) since oral candidiasis is a predisposing factor for pharyngeal involvement [12-15].

2.2.2. Virologic Failure. The main outcome of interest for this study was VF, defined as having plasma viral load $\geq 10,000$ copies $/ \mathrm{mL}$ after the first six months of therapy. VF was dichotomized into yes or no. A cut-off of 10,000 copies/mL was used for defining viral failure since values of more than 10,000 copies $/ \mathrm{mL}$ have been associated with subsequent clinical progression and appreciable CD4 cell count decline [6].

2.3. Statistical Analyses. Frequency counts and percentages were used to describe the demographic profile of the study sample.

For this study, three sets of inferential analyses were performed.

(1) The virologic status of the patients in the baseline $O L$ present group was compared with the concomitant proportions in the baseline OL absent group by means of Chi-square test. The same proportions were calculated at $6,12,18$, and 24 months. The nearest value to the end of each period $(6,12,18$, and 24 months after initial visit) was recorded.

(2) Survival analyses:

(a) Kaplan-Meier analysis was used to estimate time to VF after the first six months of therapy. Patients were censored after 24 months of followup, and for those who did not reach the study endpoint or die, data were censored at the date of their last clinic visit. To compare 
the baseline OL present and baseline OL absent groups with respect to time to VF after the first six months of therapy, log-rank statistics were used to test for a significant difference in the hazard ratio (HR) among the groups. Stratified analysis for the subgroups: viral load $<10,000$ copies $/ \mathrm{mL}$ versus viral load $\geq 10,000$ copies $/ \mathrm{mL}$ was also performed. The purpose of this stratified analysis was to test whether the relationships between the independent variable (presence/absence of baseline OL) and time to VF were identical for each subgroup. In each substratification the survival curves were compared for statistical significance.

(b) Cox proportional hazard multivariate models were used to evaluate the risk factors (presence of baseline OL, baseline demographic, and other baseline HIV-related factors) for time to VF. Correlation analyses were done to identify potential multicolinearity between independent variables. Variables that were statistically significant at $P<0.05$ on bivariate analysis and those that are known to be associated with VF in previous studies were included in the multivariable model.

(3) To evaluate the potential role of OL as clinical marker, sensitivity, specificity, positive predictive values, and negative predictive values were calculated at baseline, $6,12,18$, and 24 months. Sensitivity is the probability that an individual having VF will be OL-positive at the same visit. This was computed in the study by dividing the number of true positive cases (concurrently, OLpositive and VF-positive) by the number of patients with VF (as the "gold standard"). Specificity is the probability that an individual not having VF will be OL-negative at the same visit. This probability was computed by dividing the number of true negative cases (concurrently, OL-negative and VF-negative) by the number of patients without VF (as the "gold standard"). Positive predictive values were calculated from the subgroup of patients with VF and the concurrent OL, divided by the total number of patients from this cohort (having or not having $\mathrm{VF}$ ) presenting with OL. Negative predictive values were calculated from the subgroup of patients without VF and no OL, divided by the total number of patients from this cohort (having or not having VF) not presenting with any OL. Positive predictive values and negative predictive values were calculated at baseline, $6,12,18$, and 24 months. Statistical Package for Social Sciences software (SPSS 18 for Windows; SPSS Inc., IL, USA) was used for all of the statistical analyses.

\section{Results}

3.1. Descriptives. The study sample consisted primarily of men $(75.3 \%)$ with mean age of 39 years $(S D \pm 9.2)$. Half of the sample was African-American (49.9\%). Almost one-half
TABLE 1: Orofacial lesions associated with HIV/AIDS in adults (classification recommended by the EC Clearinghouse on Oral Problems Related to HIV Infection and WHO Collaborating Centre on Oral Manifestations of the Human Immunodeficiency Virus).

Lesions strongly associated with HIV infection

(i) Candidiasis

(a) Erythematous

(b) Pseudomembranous

(ii) Hairy leukoplakia

(iii) Kaposi's sarcoma

(iv) Non-Hodgkin's lymphoma

(v) Periodontal disease

(a) Linear gingival erythema

(b) Necrotizing (ulcerative) gingivitis

(c) Necrotizing (ulcerative) periodontitis

Lesions less commonly associated with HIV infection

(i) Bacterial infections

(a) Mycobacterium avium-intracellulare

(b) Mycobacterium tuberculosis

(ii) Melanotic hyperpigmentation

(iii) Necrotizing (ulcerative) stomatitis

(iv) Salivary gland disease

(a) Dry mouth due to decreased salivary flow rate

(b) Unilateral or bilateral swelling of the major

(v) Thrombocytopenic purpura

(vi) Ulceration NOS (not otherwise specified)

(vii) Viral infections

(a) Herpes simplex virus

(b) Human papilloma virus (wart-like lesions)

(1) Condyloma acuminatum

(2) Focal epithelial hyperplasia

(c) Verruca vulgaris

(1) Varicella zoster virus

(2) Herpes zoster

(3) Varicella

Lesions seen in HIV infection

(i) Bacterial infections

(a) Actinomyces israelii

(b) Escherichia coli

(c) Klebsiella pneumoniae

(ii) Cat-scratch disease

(iii) Drug reactions (ulcerative, erythema multiforme, lichenoid, and toxic epidermolysis)

(iv) Epithelioid (bacillary) angiomatosis

(v) Neurologic disturbances
(a) Facial palsy
(b) Trigeminal neuralgia 
TABLE 1: Continued.

(i) Candidiasis

(a) Erythematous

(b) Pseudomembranous

(ii) Hairy leukoplakia

(iii) Kaposi's sarcoma

(iv) Non-Hodgkin's lymphoma

(v) Periodontal disease
(a) Linear gingival erythema
(b) Necrotizing (ulcerative) gingivitis
(c) Necrotizing (ulcerative) periodontitis

(vi) Fungal infection other than candidiasis
(a) Cryptococcus neoformans
(b) Geotrichum candidum
(c) Histoplasma capsulatum
(d) Mucoraceae (mucormycosis/zygomycosis)
(e) Aspergillus flavus

(vii) Recurrent aphthous stomatitis

(viii) Viral infections

(a) Cytomegalo virus

(b) Molluscum contagiosum

(47.2\%) of the individuals in the study were ART-experienced before starting a new regimen at the 1917 clinic. The route of HIV transmission was self-reported as men having sex with men for $42.3 \%$ of the subjects, men having sex with women for $27.8 \%$, intravenous drug use for $8.6 \%$, and unknown routes for $21.2 \%$. Thirty-six patients $(4.8 \%)$ died during the study period. The overall prevalence of OL during the 2-year follow-up period was $35.8 \%$. In the sample population, the most common OL detected was OPC (74.9\%), followed by oral herpes $(10.4 \%)$, oral ulcer $(9.1 \%)$, and oral hairy leukoplakia (3.2\%). Kaposi's sarcoma, undetermined stomatitis, and salivary gland enlargement together represented $2.4 \%$.

3.2. Oral Lesions Mirroring Viral Load Burden. At baseline and 12 months, individuals with OL were more likely to have higher viral load values when compared to patients without OL at the baseline visit $(P<0.05)$. After the first six months of ART, this same association was only found at the 12-month time point $(P<0.05)$ (Table 2$)$.

3.3. Time to Virologic Failure. Based on the retrospectively collected data, 289 patients (42.7\%) had VF after the first six months of ART. The mean time to VF among the overall sample was 18 months $(95 \%$ CI 18,19$)$. The mean time to VF among patients with baseline OL was 17 months (95\% CI $16,18)$, while the mean time among patients without baseline OL was 19 months $(95 \%$ CI 18, 20) and the difference between these times was statistically significant $(\log$-rank, $P<0.01)$ (Figure 1). Statistically significant difference between patients having baseline OL and those without baseline OL in the

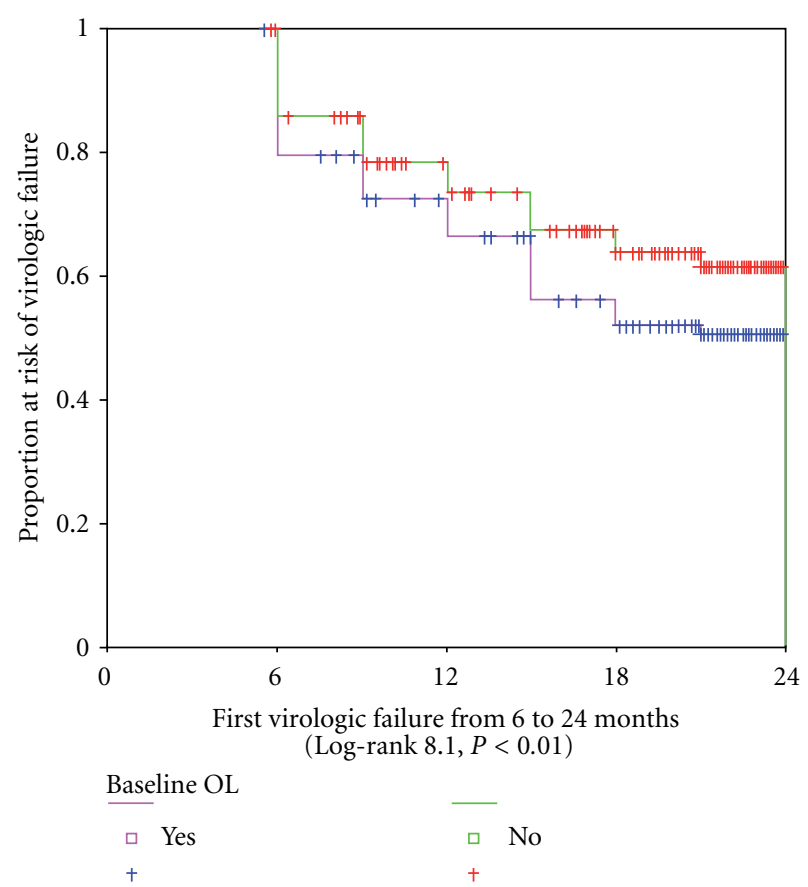

FIGURE 1: Time to virologic failure ( $\geq 10,000$ copies $/ \mathrm{mL})$ compared between oral lesion group and no-oral lesion group.

stratified analysis (baseline viral load $<10,000$ copies $/ \mathrm{mL}$ group versus baseline viral load $\geq 10,000$ copies/mL group) (log-rank, $P<0.05$ ) was also found.

3.4. Baseline Factors Associated with Time to Virologic Failure. When modeling the Cox proportional hazard model, patients who were African-American (HR 1.356; 95\% CI = 1.045-0.759), were ART-experienced (HR 2.298; 95\% CI = 1.743-3.030), had a diagnosis of mental disorder (HR 1.410; 95\% CI $=1.078-1.843)$, and had high baseline viral load (HR $2.82 ; 95 \% \mathrm{CI}=1.661-3.137$ ), were more likely to have VF after the first six months of HIV treatment. Baseline CD4 count below 200 cells $/ \mathrm{mm}^{3}$ showed low hazard ratio for VF (HR 0.715 ; 95\% CI $=0.541-0.945$ ) (Table 3 ).

3.5. Positive Predictive Value of Oral Lesions. Data presented in Table 4 assessed the value of OL in monitoring the efficacy of ART in the sample population. Overall, the sensitivity of OL was very low (except at baseline) and specificity was high for VF. OL had a strong positive predictive values for VF, especially at baseline, 12, and 24 months, and a strong negative predictive value for VF at all time points except at baseline.

\section{Discussion}

This study was an attempt to establish OL as clinical markers for VF. The prevalence of OL in the study sample was $35.8 \%$, which is similar to findings of previous studies in highresource settings $[8,10]$. However, $85 \%$ of the diagnoses of $\mathrm{OL}$ were at the baseline visit. After initiating therapy, 
TABLE 2: HIV patients initiating ART with or without oral lesions (OL) by concurrent virologic status at baseline, 6, 12, 18, and 24 months $(n=744)$.

\begin{tabular}{|c|c|c|c|c|}
\hline Status in time & $\begin{array}{c}\text { Without OL } \\
n(\%)\end{array}$ & $\begin{array}{l}\text { With OL } \\
n(\%)\end{array}$ & $\begin{array}{c}\text { All patients } \\
n(\%)\end{array}$ & $P$-value \\
\hline Baseline $\mathrm{VL}^{1}$ (copies/mL) & OL at baseline & OL at baseline & & \multirow{3}{*}{$<0.01$} \\
\hline$<10,000$ & $212(39.8)$ & $22(15.9)$ & $234(34.9)$ & \\
\hline$\geq 10,000$ & $320(60.2)$ & $116(84.1)$ & $436(65.1)$ & \\
\hline 6-month VL (copies/mL) & OL at 6 months & OL at 6 months & & \multirow{3}{*}{0.18} \\
\hline$<10,000$ & $283(72.8)$ & $6(54.5)$ & $289(72.3)$ & \\
\hline$\geq 10,000$ & $106(27.2)$ & $5(45.5)$ & $111(27.8)$ & \\
\hline 12-month VL (copies/mL) & OL at 12 months & OL at 12 months & & \multirow{3}{*}{$<0.01^{2}$} \\
\hline$<10,000$ & $261(78.4)$ & $1(20.0)$ & $262(77.5)$ & \\
\hline$\geq 10,000$ & $72(21.6)$ & $4(80.0)$ & $76(22.5)$ & \\
\hline 18-month VL (copies/mL) & OL at 18 months & OL at 18 months & & \multirow{3}{*}{$0.62^{2}$} \\
\hline$<10,000$ & $252(77.3)$ & $4(66.7)$ & $256(77.1)$ & \\
\hline$\geq 10,000$ & $74(22.7)$ & $2(33.3)$ & $76(22.9)$ & \\
\hline 24-month VL (copies/mL) & OL at 24 months & OL at 24 months & & \multirow{3}{*}{$0.06^{2}$} \\
\hline$<10,000$ & $212(75.4)$ & $0(0.0)$ & $212(74.9)$ & \\
\hline$\geq 10,000$ & $69(24.6)$ & $2(100.0)$ & $71(25.1)$ & \\
\hline
\end{tabular}

TABLE 3: Factors associated with time to virologic failure among 588 patients (156 cases had missing values) ${ }^{1}$.

\begin{tabular}{|c|c|c|c|c|}
\hline \multirow{2}{*}{ Predictors } & \multirow{2}{*}{$\begin{array}{l}\text { Bivariate analyses } \\
\text { Crude } \mathrm{HR}^{2}\end{array}$} & \multicolumn{3}{|c|}{ Multivariate analyses } \\
\hline & & Adj $\mathrm{HR}^{2}$ & $95 \% \mathrm{CI}^{3}$ & $P$ value \\
\hline \multicolumn{5}{|l|}{ Race/ethnicity } \\
\hline \multicolumn{5}{|l|}{ Caucasian (ref) } \\
\hline African-American & 1.293 & 1.356 & $1.045-1.759$ & $P<0.05$ \\
\hline Other & 0.757 & 0.943 & $0.343-2.594$ & $P=0.91$ \\
\hline \multicolumn{5}{|c|}{ Antiretroviral therapy naïve } \\
\hline \multicolumn{5}{|c|}{ Naïve (ref) } \\
\hline Experienced & 0.585 & 2.298 & $1.743-3.030$ & $P<0.01$ \\
\hline \multicolumn{5}{|c|}{ History of substance abuse } \\
\hline \multicolumn{5}{|c|}{ No (ref) } \\
\hline Yes & 1.451 & 1.311 & $0.982-1.751$ & $P=0.07$ \\
\hline \multicolumn{5}{|c|}{ Affective mental health disorder } \\
\hline \multicolumn{5}{|c|}{ No (ref) } \\
\hline Yes & 1.319 & 1.410 & $1.078-1.843$ & $P<0.01$ \\
\hline \multicolumn{5}{|l|}{ Baseline CD4 } \\
\hline \multicolumn{5}{|l|}{$\geq 200$ cell/uL (ref) } \\
\hline$<200$ cell/uL & 0.825 & 0.715 & $0.541-0.945$ & $P<0.05$ \\
\hline \multicolumn{5}{|l|}{ Baseline $\mathrm{VL}^{4}$} \\
\hline \multicolumn{5}{|c|}{$<10,000$ copies/mL (ref) } \\
\hline$\geq 10,000$ copies $/ \mathrm{mL}$ & 1.352 & 2.282 & $1.661-3.137$ & $P<0.01$ \\
\hline
\end{tabular}

${ }^{1}$ Only variables that were retained by the adjusted model were presented in this table. Variables included in the crude model: baseline OL, age, sex, race, insurance status, ART naïve/experienced, transmission risk, tobacco use, alcohol abuse, substance abuse, mental health disorder, baseline CD4, and baseline VL. ${ }^{2}$ Hazard ratios, significant association $(P<0.05) .{ }^{3}$ Confidence interval. ${ }^{4}$ VL: viral load.

only an association of VF and OL at one time point was observed. However, at all follow-up time points few OL were recorded in the study population. Previous reports support that the presence of OL is strongly associated with immune suppression but modestly associated with high viral burden in patients on ART $[16,17]$.
The overall prevalence of patients having VF within the two-year follow-up period was high (42.7\%). Similar findings have been shown by different study groups in the country, where several factors such as late diagnosis, challenges in accessing care, and transitory retention conspire to keep the collective viral load high in other US cohorts [18-20]. 
TABLE 4: Ability of HIV-related oral lesions to predict a concurrent virologic failure (viral load $\geq 10,000$ copies) at baseline, 6 months, 12 months, 18 months, and 24 months.

\begin{tabular}{lcccc}
\hline Oral lesion & $\begin{array}{c}\text { Sensitivity } \\
(\%)\end{array}$ & $\begin{array}{c}\text { Specificity } \\
(\%)\end{array}$ & $\mathrm{PPV}^{1}(\%)$ & $\begin{array}{c}\mathrm{NPV}^{2} \\
(\%)\end{array}$ \\
\hline Baseline & 26.6 & 90.6 & 84.1 & 39.8 \\
6 months & 4.5 & 97.9 & 45.5 & 72.8 \\
12 months & 5.3 & 99.6 & 80.0 & 78.4 \\
18 months & 2.6 & 98.4 & 33.3 & 77.3 \\
24 months & 2.8 & 100.0 & 100.0 & 75.4 \\
\hline${ }^{1}$ PPV: positive predictive value. ${ }^{2}$ NPV: negative predictive value.
\end{tabular}

In this population, the VF rate increased rapidly in the first year. WHO guidelines for ART therapy [11] explain that patients should be closely monitored during the first six months on ART as some patients may not respond initially and present clinical complications in some instances. This is particularly visible in patients who started therapy with advanced HIV infection. Therefore, health care professionals should be mindful that a VF could also be explained by the fact that it takes time to suppress the viral replication and boost the immune system once the patient initiates the therapy.

Presence of $\mathrm{OL}$ at baseline was predictive of a shorter time to VF in this study (17 months for patients with baseline OL versus 19 months for patients without baseline OL). While these results were statistically significant, they lack clinical importance. Analyses stratifying patients by baseline viral load $(<10,000$ copies $/ \mathrm{mL}$ versus $\geq 10,000$ copies $/ \mathrm{mL})$ revealed that regardless of baseline viral load values, the presence of baseline OL predicted a shorter time to VF. However, exploratory analysis using different cut-offs for VF ( $\geq 5,000$ copies $/ \mathrm{mL}$ and $\geq 1,000$ copies $/ \mathrm{mL}$ ) showed no statistical differences among patients with baseline OL and those patients without baseline OL.

Cox analyses allowed us to evaluate how sociodemographic characteristics and other factors may be associated with time to VF. Presence of baseline OL was not associated with shorter time to VF in these multivariate models. Other factors that were associated with VF in these models were race, exposure to ART, substance use, mental illness, and higher viral load at baseline.

This study reported a low sensitivity for OL as a predictor of VF. Patton [17] reported a higher value for sensitivity (58.2\%) in a mixed cohort (adults and children) of 606 HIV/AIDS patients at the University of North Carolina. In this longitudinal observational study, viral load data were obtained within 3 months before or after the oral examination. Perhaps, the accuracy of OL identification in this cohort was higher compared to our cohort because the oral assessment in the North Carolina Study was conducted by an oral medicine-trained examiner. While a high sensitivity is desirable for any new diagnostic test, the goal for using OLs as clinical markers is to ensure that patients who are identified by the presence of OL are very likely to have VF, that is, high positive predictive values, even if the sensitivity is low and many failing patients are not identified.
Sensitivity, specificity, positive predictive values, and negative predictive values of OPC for immune and viral failure at baseline and 6 months have been reported by Miziara and Weber [21] in a Brazilian cohort of 124 HIVinfected patients on ART. The Brazilian study found high sensitivity and negative predictive values at baseline and 6 months. However, their specificity and positive predictive values were lower compared to our findings. In this cohort, OL diagnosis was performed by the same physician.

As other studies have suggested [17, 21], high specificity of OL provides value in "ruling in" good immune function and attainment or maintenance of viral suppression when OLs are not present. Among individuals who are on ART, OL may be a harbinger of failure caused by nonadherence or development of drug-resistant mutations.

Unfortunately, results in this study population did not fully support the hypothesis of OL as an accurate indicator of concurrent VF or predictor for VF. In low-resource settings where the gold standards for monitoring ART (CD4 and viral load) are scarce or not available, the search for an accurate surrogate marker remains a priority. In addition, these findings should be interpreted with caution. As a retrospective study from a single HIV cohort, these results may not be generalizable. An important limitation was that not all of the patients in the cohort had a complete CD4 count and viral load determination either at baseline, 6, 18 , and 24 months. Even more, $42 \%$ of the sample was lost to followup by the end of the study period. This was not desirable, but somehow expected due the fact that poor retention in HIV care has become a critical challenge in ART national programs since the last few years [2226].

In addition, data on use of antifungal therapy was not available in the database and this could have a substantial impact on subsequent prevalence of OPC. It is likely that the antifungals may have had a significant impact on this particular OL since most of the episodes of OPC were reported during the first 6 months on therapy. The fact that OL diagnosis was not quality assured for inter- or intraexaminer differences could have led to an underestimation of OL as has been previously reported [27]. At the 1917 Clinic, as in many other HIV clinics around the US, it is the treating general clinician, not a dental health professional, who performs the intraoral examination most of the time. Therefore, there is potential for reporting bias in this study since not all clinicians were trained specifically in the diagnosis of OL and clinician reporting data was not available for analysis.

The strengths of this study include a large sample of nearly 750 patients on ART and the fact that the study population was evenly distributed by levels of CD4 count and viral load, which offered the opportunity to explore OL in relation to VF.

In conclusion, this study did not find clear associations of OL and VF in a population of HIV-infected patients initiating therapy. Further studies would be indicated prior to using these criteria as a single clinical surrogate for VF. 


\section{Acknowledgments}

The authors would like to thank all of the members of the University of Alabama at Birmingham HIV Outpatient Clinic (1917 Clinic) Database Group for their assistance with data access. Partial results of this study were presented in July 2011 at the 6th International AIDS Society Conference on HIV Pathogenesis, Treatment and Prevention (IAS 2011) in Rome, Italy.

\section{References}

[1] W. Birnbaum, T. A. Hodgson, P. A. Reichart, W. Sherson, S. W. Nittayannanta, and T. E. Axell, "Prognostic significance of HIV-associated oral lesions and their relation to therapy," Oral Diseases, vol. 8, no. 2, pp. 110-114, 2002.

[2] J. S. Greenspan, "Sentinels and signposts: the epidemiology and significance of the oral manifestations of HIV disease," Oral Diseases, vol. 3, no. 1, pp. S13-S17, 1997.

[3] V. Margiotta, G. Campisi, S. Mancuso, V. Accurso, V. Abbadessa, and P. Giaccone, "HIV infection: oral lesions, $\mathrm{CD} 4^{+}$cell count and viral load in an italian study population," Journal of Oral Pathology and Medicine, vol. 28, no. 4, pp. 173-177, 1999.

[4] M. M. Coogan, J. Greenspan, and S. J. Challacombe, "Oral lesions in infection with human immunodeficiency virus," Bulletin of the World Health Organization, vol. 83, no. 9, pp. 700-706, 2005.

[5] T. A. Hodgson, D. Greenspan, and J. S. Greenspan, "Oral lesions of HIV disease and HAART in industrialized countries," Advances in Dental Research, vol. 19, no. 1, pp. 57-62, 2006.

[6] Guideline for the use of antiretroviral agents in HIV-1 infected aults and adolescents. Developed by the DHHS Panel on Antiretroviral Guidelines for Adults and Adolescents-A working Group of the Office of AIDS Research Advisory Council, 2006.

[7] J. A. C. Sterne, M. A. Hernán, B. Ledergerber et al., "Longterm effectiveness of potent antiretroviral therapy in preventing AIDS and death: a prospective cohort study," The Lancet, vol. 366, no. 9483, pp. 378-384, 2005.

[8] A. R. Tappuni and G. J. P. Fleming, "The effect of antiretroviral therapy on the prevalence of oral manifestations in HIVinfected patients: a UK study," Oral Surgery, Oral Medicine, Oral Pathology, Oral Radiology, and Endodontics, vol. 92, no. 6, pp. 623-628, 2001.

[9] L. A. Gaitán-Cepeda, M. Martínez-González, and A. CeballosSalobreña, "Oral candidosis as a clinical marker of immune failure in patients with HIV/AIDS on HAART," AIDS Patient Care and STDs, vol. 19, no. 2, pp. 70-77, 2005.

[10] L. L. Patton, R. McKaig, R. Strauss, D. Rogers, and J. J. Eron, "Changing prevalence of oral manifestations of human immuno-deficiency virus in the era of protease inhibitor therapy," Oral Surgery, Oral Medicine, Oral Pathology, Oral Radiology, and Endodontics, vol. 89, no. 3, pp. 299-304, 2000.

[11] WHO, Antiretroviral Therapy for HIV Infection in Adults and Adolescents: Recommendations for a Public Health Approach, Edited by WHO, World Health Organization Guidelines, 2006.

[12] Candidiasis, In: Dental Issues: Oral Cancer Foundation, 2010, http://www.oralcancerfoundation.org/dental/candida.htm.

[13] M. Ravera, A. Reggiori, A. M. Agliata, and R. P. Rocco, "Evaluating diagnosis and treatment of oral and esophageal candidiasis in Ugandan AIDS patients," Emerging Infectious Diseases, vol. 5, no. 2, pp. 274-277, 1999.
[14] Candidiasis, "Oral and Esophageal," in Guide for HIV/AIDS Clinical Care: HIV/AIDS Bureau, U.S. Department of Health and Human Services, Health Resources, and Services Administration, 2011.

[15] P. G. Pappas, C. A. Kauffman, D. Andes et al., "Clinical practice guidelines for the management of candidiasis: 2009 Update by the Infectious Diseases Society of America," Clinical Infectious Diseases, vol. 48, no. 5, pp. 503-535, 2009.

[16] S. G. Revankar, S. E. Sanche, O. P. Dib, M. Caceres, and T. F. Patterson, "Effect of highly active antiretroviral therapy on recurrent oropharyngeal candidiasis in HIV-infected patients," AIDS, vol. 12, no. 18, pp. 2511-2513, 1998.

[17] L. L. Patton, "Sensitivity, specificity, and positive predictive value of oral opportunistic infections in adults with HIV/AIDS as markers of immune suppression and viral burden," Oral Surgery, Oral Medicine, Oral Pathology, Oral Radiology, and Endodontics, vol. 90, no. 2, pp. 182-188, 2000.

[18] C. S. Krawczyk, E. Funkhouser, J. M. Kilby, R. A. Kaslow, A. K. Bey, and S. H. Vermund, "Factors associated with delayed initiation of HIV medical care among infected persons attending a southern HIV/AIDS clinic," Southern Medical Journal, vol. 99, no. 5, pp. 472-481, 2006.

[19] M. Mascolini, Three Biggest HIV Problems in the United States: Late Testing, Late Care, Early Dropout. In: Research Initiative, Treatment Action!: AIDS Research Consortium of Houston, 2011, http://img.thebody.com/cfa/pdfs/rita_spring_summer11 .pdf $\#$ page $=5$.

[20] M. J. Mugavero, W. E. Norton, and M. S. Saag, "Health care system and policy factors influencing engagement in HIV medical care: piecing together the fragments of a fractured health care delivery system," Clinical Infectious Diseases, vol. 52, supplement 2, pp. S238-S246, 2011.

[21] I. D. Miziara and R. Weber, "Oral candidosis and oral hairy leukoplakia as predictors of HAART failure in Brazilian HIVinfected patients," Oral Diseases, vol. 12, no. 4, pp. 402-407, 2006.

[22] M. W. G. Brinkhof, F. Dabis, L. Myer et al., "Early loss of HIVinfected patients on potent antiretroviral therapy programmes in lower-income countries," Bulletin of the World Health Organization, vol. 86, no. 7, pp. 559-567, 2008.

[23] L. Gardner, G. Marks, J. Craw et al., "A low-effort clinicwide intervention improves attendance for HIV primary care," Clinical Infectious Diseases, vol. 55, no. 8, pp. 1124-1134, 2012.

[24] D. N. Burns, C. W. Dieffenbach, and S. H. Vermund, "Rethinking prevention of HIV type 1 infection," Clinical Infectious Diseases, vol. 51, no. 6, pp. 725-731, 2010.

[25] E. M. Gardner, M. P. McLees, J. F. Steiner, C. Del Rio, and W. J. Burman, "The spectrum of engagement in HIV care and its relevance to test-and-treat strategies for prevention of HIV infection," Clinical Infectious Diseases, vol. 52, no. 6, pp. 793-800, 2011.

[26] "Vital signs: HIV prevention through care and treatmentUnited States," MMWR. Morbidity and Mortality Weekly Report, vol. 60, pp. 1618-1623, 2011.

[27] J. F. Hilton, M. Alves, K. Anastos et al., "Accuracy of diagnoses of HIV-related oral lesions by medical clinicians, findings from the Women's Interagency HIV Study," Community Dentistry and Oral Epidemiology, vol. 29, no. 5, pp. 362-372, 2001. 


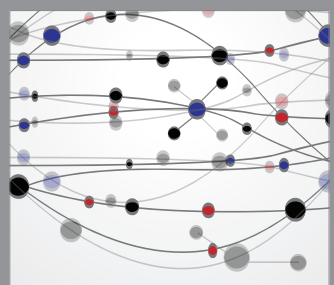

The Scientific World Journal
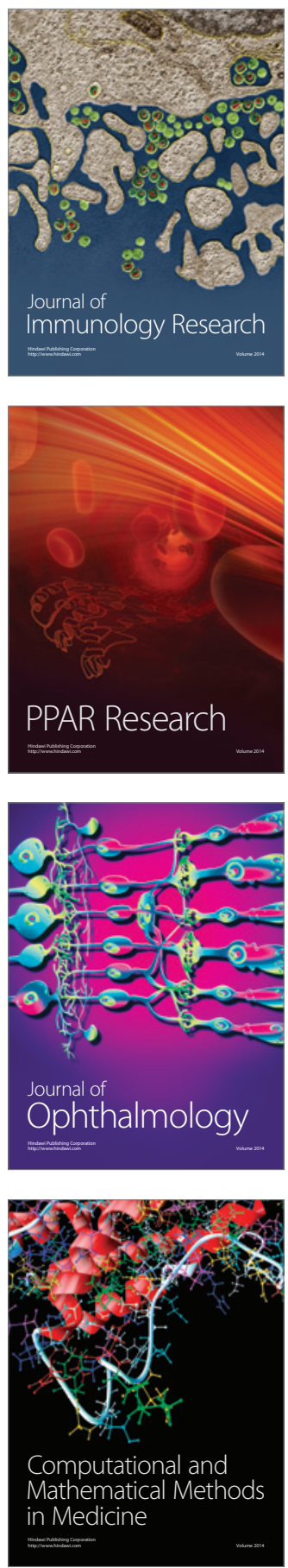

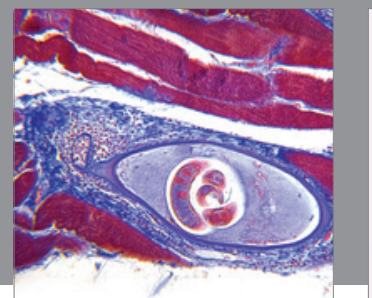

Gastroenterology

Research and Practice
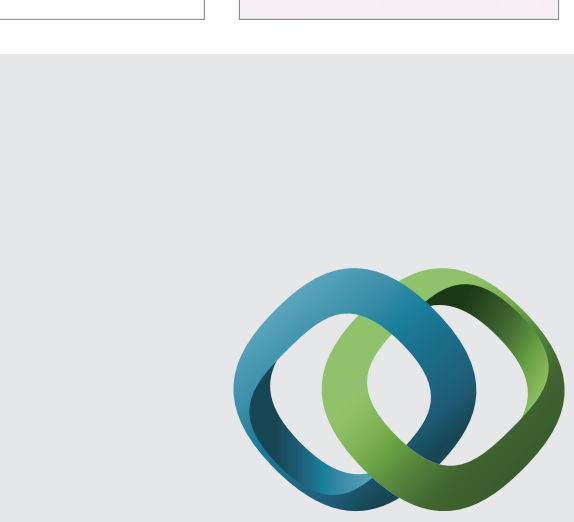

\section{Hindawi}

Submit your manuscripts at

http://www.hindawi.com
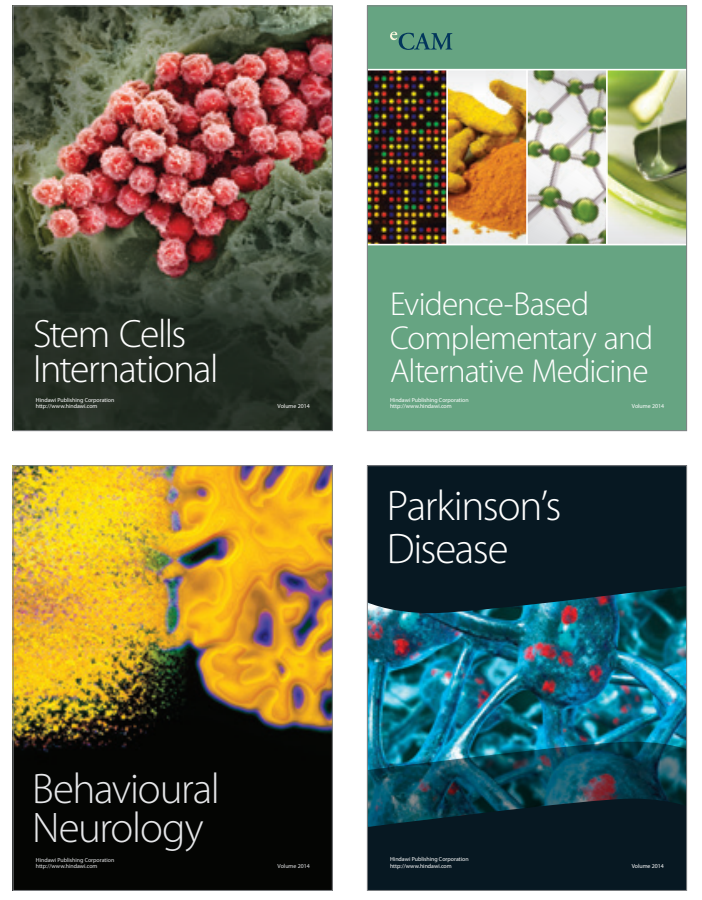
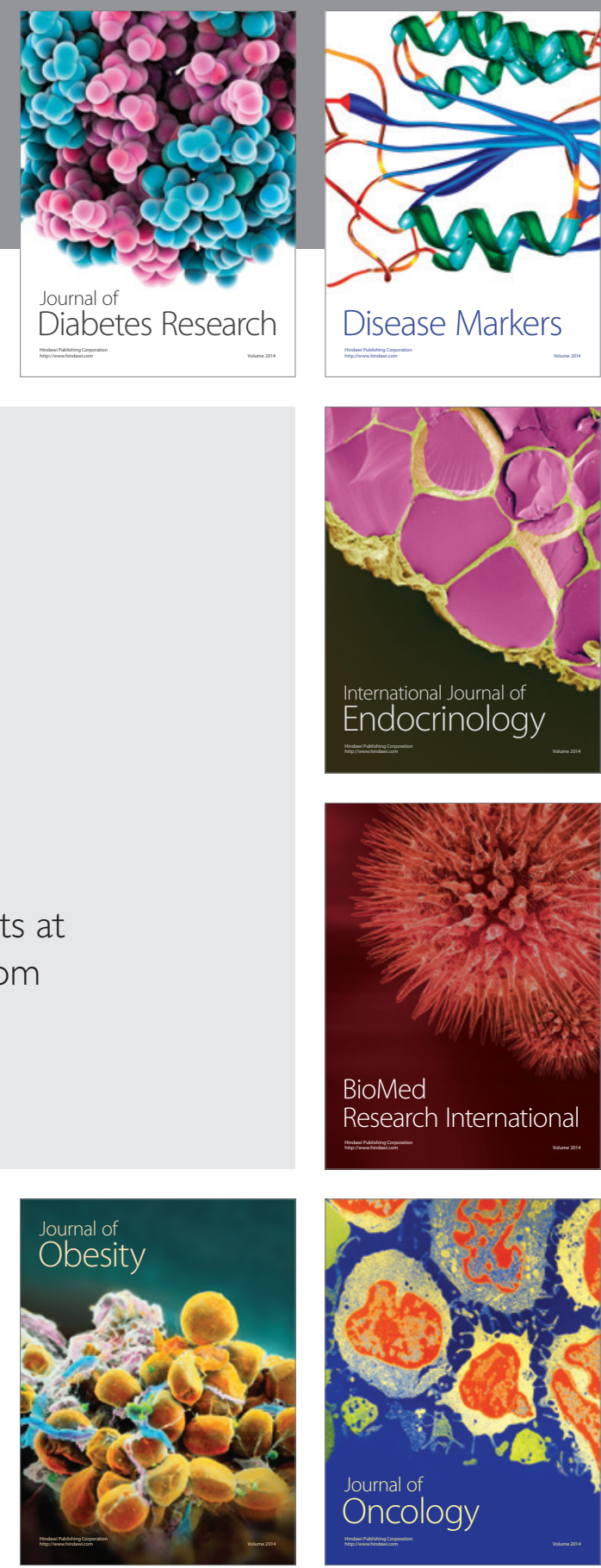

Disease Markers
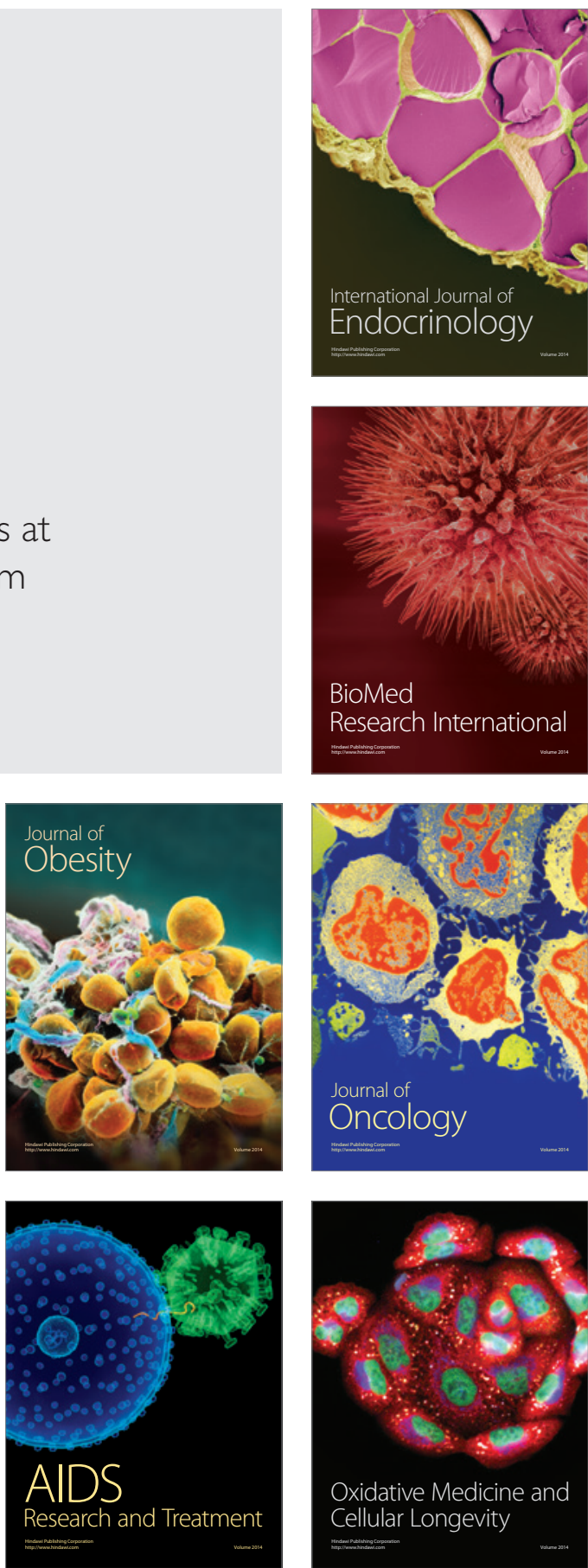